\title{
Electro-acupuncture stimulation prevents remifentanil-induced postoperative hyperalgesia by suppressing spinal microglia in rats
}

\author{
YANHU XIE, JUN MA, DI WANG, XIAOQING CHAI and CHEN GAO \\ Department of Anesthesiology, Anhui Provincial Hospital Affiliated Anhui Medical University, \\ Hefei, Anhui 230001, P.R. China
}

Received November 13, 2017; Accepted March 23, 2018

DOI: $10.3892 /$ etm.2018.6161

\begin{abstract}
The aim of the present study was to assess the effect of electro-acupuncture (EA) stimulation on remifentanil-induced postoperative hyperalgesia (RIPH) and the possible involvement of spinal microglia suppression. A model of RIPH was established using adult male Sprague-Dawley rats by administration of remifentanil at $0.08 \mathrm{mg} / \mathrm{kg}$ intravenously for $60 \mathrm{~min}$. The Huantiao and Yanglingquan acupoints were stimulated continuously by EA ( $2 \mathrm{~Hz}, \sim 1 \mathrm{~mA}$ ) for 90 min from before paw incision to the end of remifentanil administration. Sham acupoints were stimulated by EA in the sham group. Paw withdrawal threshold (PWT) and paw thermal withdrawal latency (PWL) were determined. Cluster of differentiation (CD) $11 \mathrm{~b}$, tumor necrosis factor (TNF)- $\alpha$, interleukin (IL)- $1 \beta$ and IL- 6 levels in spinal cord samples were measured using immunohistochemistry and ELISA. PWT and PWL values were decreased following the administration of remifentanill; however, following EA, PWT and PWL values increased compared with the sham group $(\mathrm{P}<0.05)$, indicating that EA alleviates remifentanil-induced RIPH. CD11b, TNF- $\alpha$, IL-1 $\beta$ and IL- 6 levels were increased following remifentanil administration and these effects were counteracted by EA (all $\mathrm{P}<0.05$ ). In the sham group, no significant differences were observed in PWT and PWL values or CD11b, TNF- $\alpha$, IL-1 $\beta$ and IL- 6 levels compared with the control group, suggesting that EA was responsible for the
\end{abstract}

Correspondence to: Dr Xiaoqing Chai, Department of Anesthesiology, Anhui Provincial Hospital Affiliated Anhui Medical University, 17 Lujiang Road, Hefei, Anhui 230001, P.R. China

E-mail:2660747430@qq.com

Abbreviations: EA, electro-acupuncture; NMDARs, $\mathrm{N}$-methyl-D-aspartate receptors; NS, normal saline; OIH, opioid-induced hyperalgesia; PWL, paw thermal withdrawal latency; PWT, paw withdrawal threshold; RF, remifentanil; RF/EA, remifentanil and electro-acupuncture; RF/EA-sham, remifentanil and sham acupuncture; RIPH, remifentanil-induced postoperative hyperalgesia

Key words: electro-acupuncture, remifentanil, postoperative hyperalgesia, pro-inflammatory cytokines, microglia reduction in CD11b and pro-inflammatory cytokine expression following remifentanil administration. The results of the present study demonstrated that EA at the Huantiao and Yanglingquan acupoints may reduce remifentanil-induced postoperative hyperalgesia, likely by inhibiting spinal microglia via reduction of CD11b and pro-inflammatory cytokine expression. However, these results are preliminary and require further validation.

\section{Introduction}

Remifentanil (RF) is widely used in general anesthesia as a potent ultra-short-acting opioid $\mu$ receptor agonist, with a rapid onset and short action time $(1,2)$. RF is able to cause opioid-induced hyperalgesia $(\mathrm{OIH})$, enhancing pain sensitivity and making it more difficult to manage postoperative pain $(3,4)$. It is known that hyperalgesia induced by a high-dose (0.40-0.2 $\mu \mathrm{g} / \mathrm{kg} / \mathrm{min})$ of RF results in increased morphine consumption after surgery $(5,6)$.

$\mathrm{OIH}$ is associated with decreased levels of endogenous opioid peptides and increased activation of microglia and N-methyl-D-aspartate receptors (NMDARs) (7). Microglia differentiate from spinal cord monocytes and are representative immune cells in the central nervous system that are thought to serve a role in central sensitization and pain regulation (6-8). Pro-inflammatory cytokines are associated with the activation of spinal nociceptive neurons and inflammatory pain maintenance (9). Furthermore, microglia activation is associated with a significant increase in the production of pro-inflammatory cytokines, including tumor necrosis factor (TNF)- $\alpha$, interleukin (IL) $-1 \beta$ and IL-6 $(10,11)$. Previous studies have suggested that these cytokines, in combination with the abnormal NMDAR activation, serve an important role in central sensitization and hyperalgesia in the spinal dorsal horn, possibly promoting OIH development and maintenance $(12,13)$.

Clinical studies have revealed that a number of pharmacological agents, including ketamine, propofol and nitric oxide, attenuate RF-induced hyperalgesia $(14,15)$; however, these agents may have adverse effects, including chest pain, confusion, paresthesia and hypotension, limiting their clinical application (16). Electro-acupuncture (EA) stimulation has been used for thousands of years in traditional Chinese medicine to treat acute and chronic pain with few complications (17). The efficiency and safety of EA $(18,19)$ have made 
it one of the primary complementary methods for pain treatment (9). Furthermore, EA has potential as a treatment for postoperative pain (20) and has previously been applied in postoperative analgesia $(18,21)$. In a previous study, our group demonstrated that EA reduces the analgesic dose required and ameliorates pain in patients postoperatively (22). The analgesic mechanism of EA mainly involves the release of endogenous opioid peptides, adenosine and 5-hydroxytryptamine $(23,24)$.

The effects of EA on RF-induced postoperative hyperalgesia (RIPH) remain unclear. Therefore, the aim of the present study was to assess how EA impacts RIPH and explore the underlying mechanisms. A rat model of RIPH $(25,26)$ was established and the effects of EA were assessed. The results indicated that EA prevents RIPH, likely by suppressing spinal microglia.

\section{Materials and methods}

Animals. A total of 96 adult male Sprague-Dawley rats (8-10 weeks; weighing 210-250 g) were provided by the animal center of Anhui Medical University (Hefei, China) and housed in the animal facility for 3-4 days prior to experiments. All rats were fed with a 12-h light/dark cycle at a constant room temperature of $22 \pm 2^{\circ} \mathrm{C}$ and relative humidity of $60-80 \%$. The animals had access to food and water ad libitum. The experimental protocols were approved by the Institutional Animal Experimental Ethics Committee of Anhui Medical University. All procedures were performed in accordance with the ethical standards of the Institutional Animal Care and Use Committee of Anhui Medical University.

Experimental protocol. A total of 96 rats were randomly divided into four groups ( $\mathrm{n}=24$ in each): Normal saline (NS), RF, $\mathrm{RF}+\mathrm{EA}(\mathrm{RF} / \mathrm{EA})$ and RF + sham acupuncture (RF/EA-sham). Rats were anesthetized with $30 \mathrm{mg} / \mathrm{kg}$ pentobarbital intraperitoneally. The Huantiao and Yanglingquan acupoints or corresponding sham acupoints were stimulated by EA in the RF/EA and RF/EA-sham groups, respectively, during incision and medication procedures. Plantar incisional pain was induced in each group. As appropriate, $\mathrm{NS}(0.8 \mathrm{ml} / \mathrm{h}$ for $60 \mathrm{~min})$ and RF (Yichang Renfu Pharmaceutical, Yichang, China; batch no. $6130502 ; 0.08 \mathrm{mg} / \mathrm{kg}$ at $0.8 \mathrm{ml} / \mathrm{h}$ for $60 \mathrm{~min})(25,26)$ were injected intravenously with a pump (Fig. 1).

EA procedure. Stainless steel acupuncture needles $(0.18 \times 30 \mathrm{~mm})$ were inserted $5 \mathrm{~mm}$ into the right hind leg at the Huantiao (GB30, posterior upper edge of the hip joint) and Yanglingquan (GB34, $5 \mathrm{~mm}$ below capitulum fibulae) acupoints, as previously described $(27,28)$ (Fig. 2). Stimulation was performed with a constant current pulse generator model EL-608 (NKL Electronic Products, Brusque, Brazil) for $\sim 90$ min (prior to incision until the end of RF administration). The stimuli were set as $0.3 \mathrm{msec}$ wide square waves at a frequency of $2 \mathrm{~Hz}$. Current intensity was increased in a stepwise fashion until a muscle twitch was observed $(\sim 1 \mathrm{~mA}$ at $2 \mathrm{~Hz})$ as described previously $(29,30)$. Rats in the RF/EA-sham group underwent the same procedure but needles were inserted $0.5 \mathrm{~cm}$ right to the correct acupoints (31).

Plantar incision. Plantar incision was performed as previously described by Brennan (32). Following sterilization of the

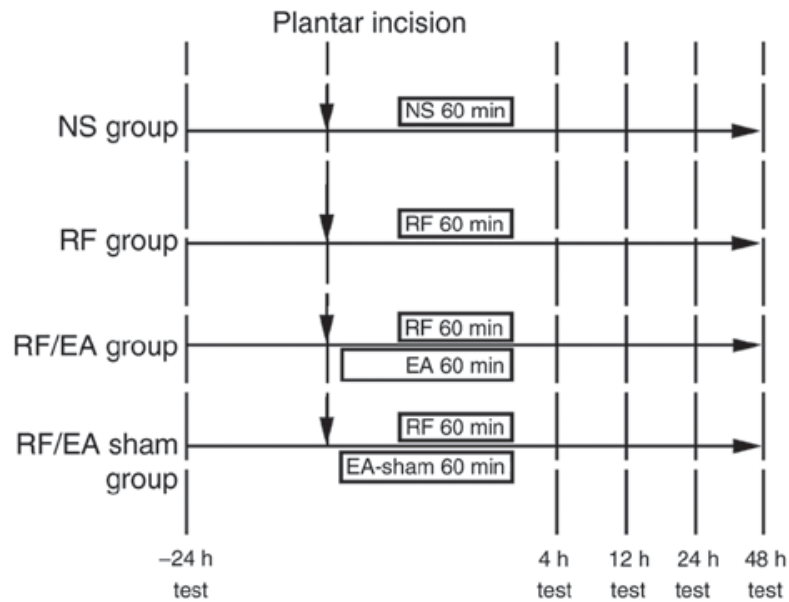

Figure 1. Schematic of experimental procedures. A plantar incision was used to create the pain model, as described by Brennan (32). EA was performed at the Huantiao and Yanglingquan acupoints in the RF/EA group and at sham acupoints in the RF/EA sham group. Stimulation was maintained for the duration of RF infusion $(0.08 \mathrm{mg} / \mathrm{kg}$ over $60 \mathrm{~min} ; 0.8 \mathrm{ml} / \mathrm{h})$. In the NS group, NS was infused at the same rate $(0.8 \mathrm{ml} / \mathrm{h})$. Paw withdrawal threshold and paw withdrawal latency were tested $24 \mathrm{~h}$ prior to infusion and at 4, 12, 24 and $48 \mathrm{~h}$ following infusion. ELISA and immunohistochemistry were performed at 4, 24 and $48 \mathrm{~h}$ following infusion. EA, electro-acupuncture; RF, remifentanil; NS, normal saline.

right hind paw with $10 \%$ iodophor (Aitefu Co., Ltd., Huai'an, China), a $1 \mathrm{~cm}$ longitudinal incision was made through the skin and fascia of the plantar aspect, starting $0.5 \mathrm{~cm}$ from the proximal edge of the heel and extending toward the toes. The plantaris muscle was elevated and incised longitudinally. The muscle origin and insertion remained intact. Following hemostasis with gentle pressure, the skin was apposed with mattress sutures. The wound was covered with an ointment containing polymyxin B, neomycin and bacitracin (Zhejiang Reachall Pharmaceutical Co., Ltd., Dongyang, China) (32).

Behavioral tests. Paw withdrawal threshold (PWT) and paw withdrawal latency (PWL) were assessed $24 \mathrm{~h}$ prior to RF infusion and at 4, 12,24 and $48 \mathrm{~h}$ following the completion of $\mathrm{RF}$ infusion. Rats were placed in individual wire cages with a mesh bottom and allowed to adapt for $60 \mathrm{~min}$ prior to testing.

Mechanical hyperalgesia was assessed using an electronic Von Frey filament (Harvard Apparatus, Holliston, MA, USA) as described by Yuan et al (33). The filament was applied vertically to the area adjacent to the wound on the right hind paw and pressure was increased until a positive response occurred. The effective pressure was then as the PWT. The test was repeated three times at 5-min intervals. A positive response was defined as clear paw withdrawal, licking or squeaking. A cutoff pressure of $60 \mathrm{~g}$ was used to prevent tissue damage.

Thermal hyperalgesia was measured using YLS-6B intelligent hotplate equipment (Zhenghua Biologic Apparatus Facilities Co., Ltd., Huaibei, China) as described by Yuan et al (33). Rats were placed on a $50^{\circ} \mathrm{C}$ hotplate until a positive response was observed. The response time was recorded as the PWL. The test was repeated three times at 10-min intervals. A positive response was defined as a clear paw withdrawal and a cutoff time of $40 \mathrm{sec}$ was used to prevent tissue damage. 


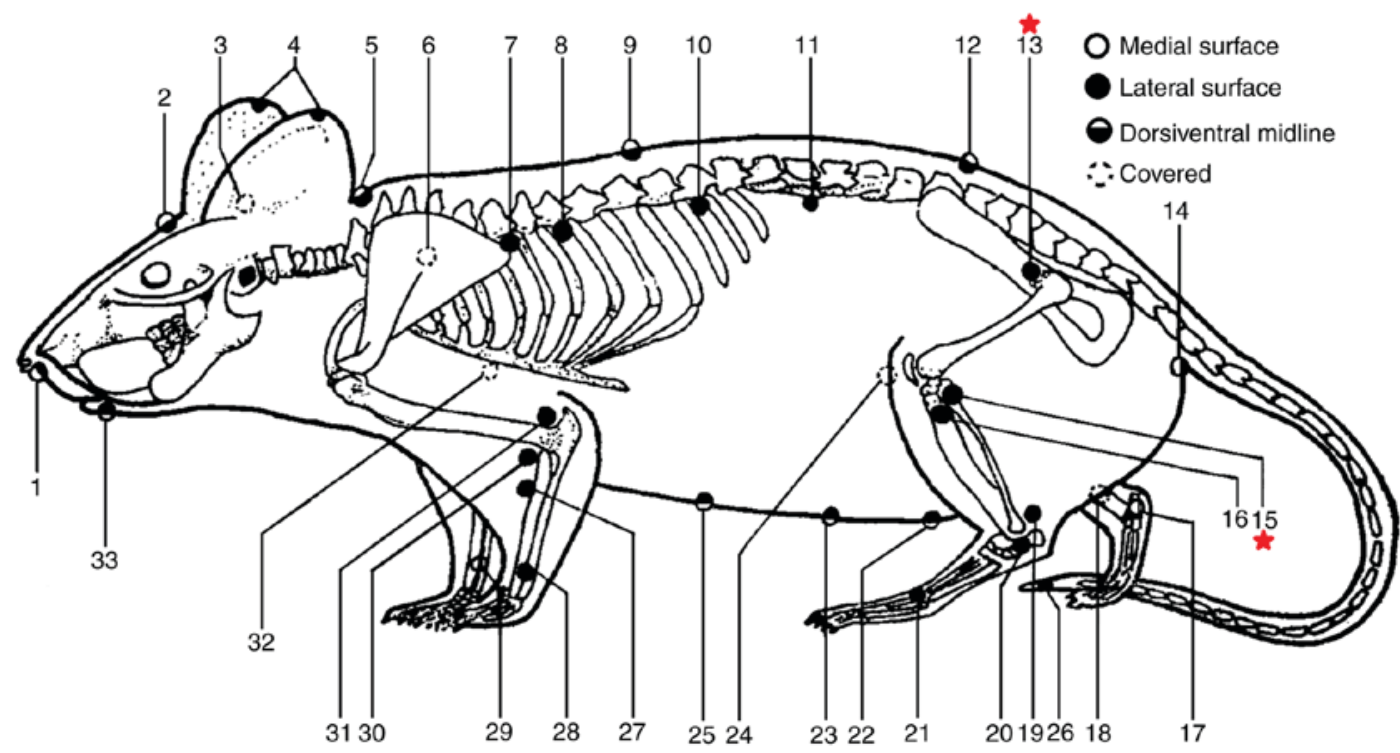

Figure 2. Equivalence of human acupoints in rats based on the Academic Department of China Association for Acupuncture and Moxibustion and the Jiangsu Institute of Traditional Chinese Medicine (28). The two acupoints used in the present study are marked with red stars. Each number represents an acupoint; the Chinese name and standard international acupuncture nomenclature are also provided. 1, Shui gou (GV26); 2, Bai hui (GV20); 3, Tian men (BL2); 4, Er jian (EX-HN6); 5, Da zhui (GV14); 6, Fei shu (UB13); 7, Xin shu (UB15); 8, Ge shu (UB17); 9, Ji zhong (GV6); 10, Pi shu (UB20); 11, Shen shu (UB23); 12, Hou hui; 13, Huan tiao (GB30); 14, Hou hai; 15, Yang ling quan (GB34); 16, Hou san 1i; 17, Zhao hai (KID6); 18, San yin jiao (SP6); 19, Gen duan; 20, Shen mai (UB62); 21, Tai chong (LIV3); 22, Guan yuan (CV4); 23, Xi qian; 24, Shen jue; 25, Zhong wan (CV12); 26, Wei jian; 27, Qian san li; 28, Wai guan (TE5); 29, Nei guan (PC6); 30, Qu chi (LI11); 31, Zhou jie; 32, Tan zhong (CV17); 33, Cheng jiang (CV24).

ELISA. Following RF infusion and behavioral tests, a total of 6 rats per group were sacrificed at each time point. TNF- $\alpha$ (cat. no. SC-52746), IL-1 $\beta$ (cat. no. SC-12742) and IL-6 (cat. no. SC-57315) levels were measured in the spinal cord at lumbar segments $\left(\mathrm{L}_{4-5}\right)$ using ELISA kits (Santa Cruz Biotechnology, Inc., Dallas, TX, USA) according to the manufacturer's protocol.

Immunohistochemistry. At 4, 24 and $48 \mathrm{~h}$ following the completion of RF infusion, following behavior testing, 2 rats were anesthetized with an intraperitoneal injection of $350 \mathrm{mg} / \mathrm{kg}$ chloral hydrate. The chest was opened and the right atrium was cut, $200 \mathrm{ml}$ saline was perfused rapidly into the left ventricular at $4^{\circ} \mathrm{C}$ and then the right atrium was perfused with $200 \mathrm{ml}$ paraformaldehyde at $4 \%$ for $6 \mathrm{~h}$. The spinal arch plate was cut off, the spinal cord was exposed, and $\mathrm{L}_{4-5}$ lumbar segments were dissected and removed. Specimens were fixed in $4 \%$ paraformaldehyde at room temperature for $24 \mathrm{~h}$ and then embedded in paraffin. Each paraffin-cut section was $4 \mathrm{~mm}$ in thickness. CD11b expression was measured in the spinal cord at lumbar segments $\left(\mathrm{L}_{4-5}\right)$ using immunostaining with the primary antibody OX-42 (cat. no. ab33827; 1:50; Abcam, Cambridge, MA, USA) at $4^{\circ} \mathrm{C}$ for $12 \mathrm{~h}$. Spinal cord sections were washed and incubated for $30 \mathrm{~min}$ at $37^{\circ} \mathrm{C}$ with horseradish peroxidase-labeled secondary antibodies (cat. no. PV-6000; 1:50; OriGene Technologies, Inc., Beijing, China). A total of 6-10 images were captured for each sample using an inverted microscope with a magnification of $\mathrm{x} 600$. The area of positive staining for CD11b was assessed using computerized morphometry (Image-Pro Plus software version 6.0; Media Cybernetics, Inc., Rockville, MD, USA).

Statistical analysis. Data are presented as the mean \pm standard deviation. Statistical analysis was performed using SPSS 16.0
(SPSS, Inc., Chicago, USA). Differences were assessed using one-way analysis of variance with a post hoc least-significant difference test for multiple comparisons. $\mathrm{P}<0.05$ was considered to indicate a statistically significant difference.

\section{Results}

EA alleviates RF-induced hyperalgesia. PWT and PWL values were similar in all groups prior to infusion and decreased gradually following infusion, with the lowest values at $24 \mathrm{~h}$ (Fig. 3). PWT and PWL values were significantly decreased in the RF and RF/EA-sham groups at 4, 12, 24 and $48 \mathrm{~h}$ following $\mathrm{RF}$ infusion compared with the NS group (all $\mathrm{P}<0.05$; Fig. 3), indicating RF-induced hyperalgesia. Higher PWT and PWL values were observed in the RF/EA group compared with the RF/EA-sham group at 4, 12, 24 and $48 \mathrm{~h}$ following RF infusion (all $\mathrm{P}<0.05$; Fig. 3). These findings suggest that EA alleviates RF-induced hyperalgesia. No significant differences in PWT and PWL values were observed between the NS and $\mathrm{RF} / \mathrm{EA}$ groups, or between the RF and RF/EA-sham groups. These results suggest suggested that RF-induced hyperalgesia was almost completely reversed following after EA treatment, while sham EA had no effect.

EA decreases CD11b levels. CD11b levels in the RF and $\mathrm{RF} / \mathrm{EA}$-sham groups were significantly increased compared with those of the NS group at 4, 24 and $48 \mathrm{~h}$ following RF infusion (all $\mathrm{P}<0.05$; Fig. 4), which indicates that RF treatment increased the amounts of spinal microglia. Compared with the RF group, CD11b levels were significantly decreased at 4, 24 and $48 \mathrm{~h}$ following $\mathrm{RF}$ infusion in the RF/EA group (all $\mathrm{P}<0.05$; Fig. 4), suggesting that EA decreased the number of spinal microglia. No significant differences in CD11b expression were observed between the NS 
A

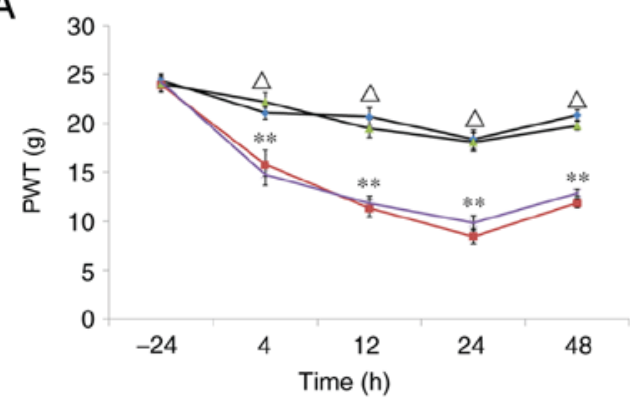

B

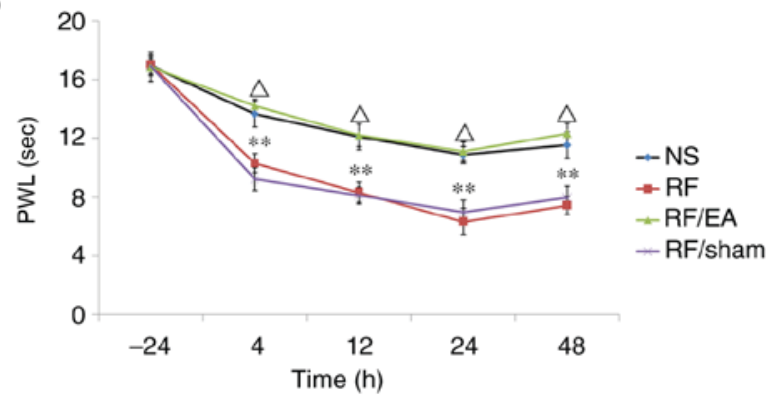

Figure 3. Effects of EA on RF-induced mechanical and thermal hyperalgesia. (A) PWT and (B) PWL were evaluated at $24 \mathrm{~h}$ prior to infusion and at $4,12,24$ and $48 \mathrm{~h}$ following infusion. ${ }^{* *} \mathrm{P}<0.05$ vs. NS and ${ }^{\wedge} \mathrm{P}<0.05$ vs. RF. EA, electro-acupuncture; PWT, paw withdrawal threshold; PWL, paw thermal withdrawal latency; NS, normal saline; RF, remifentanil.
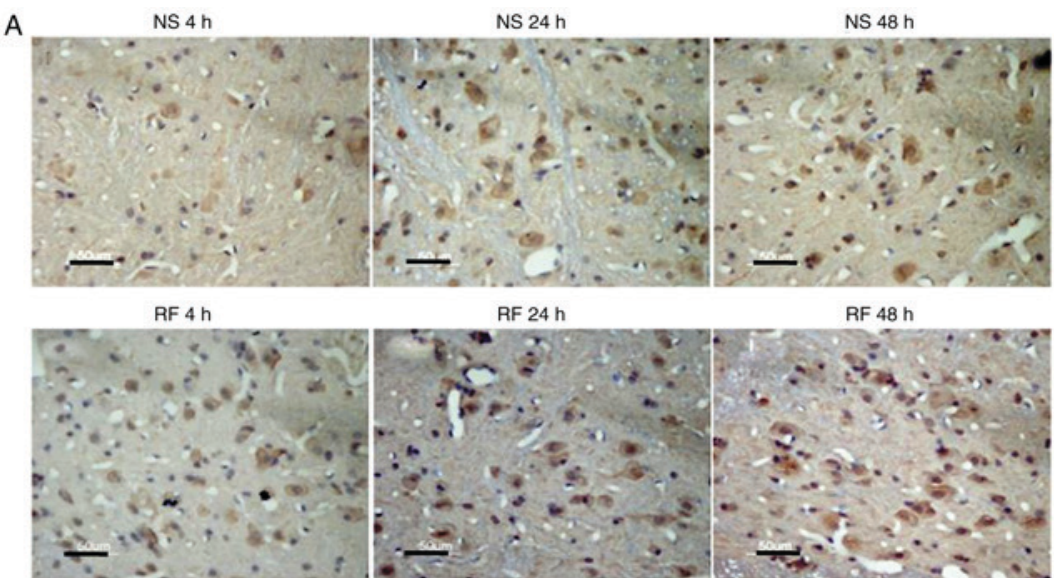

RF $48 \mathrm{~h}$
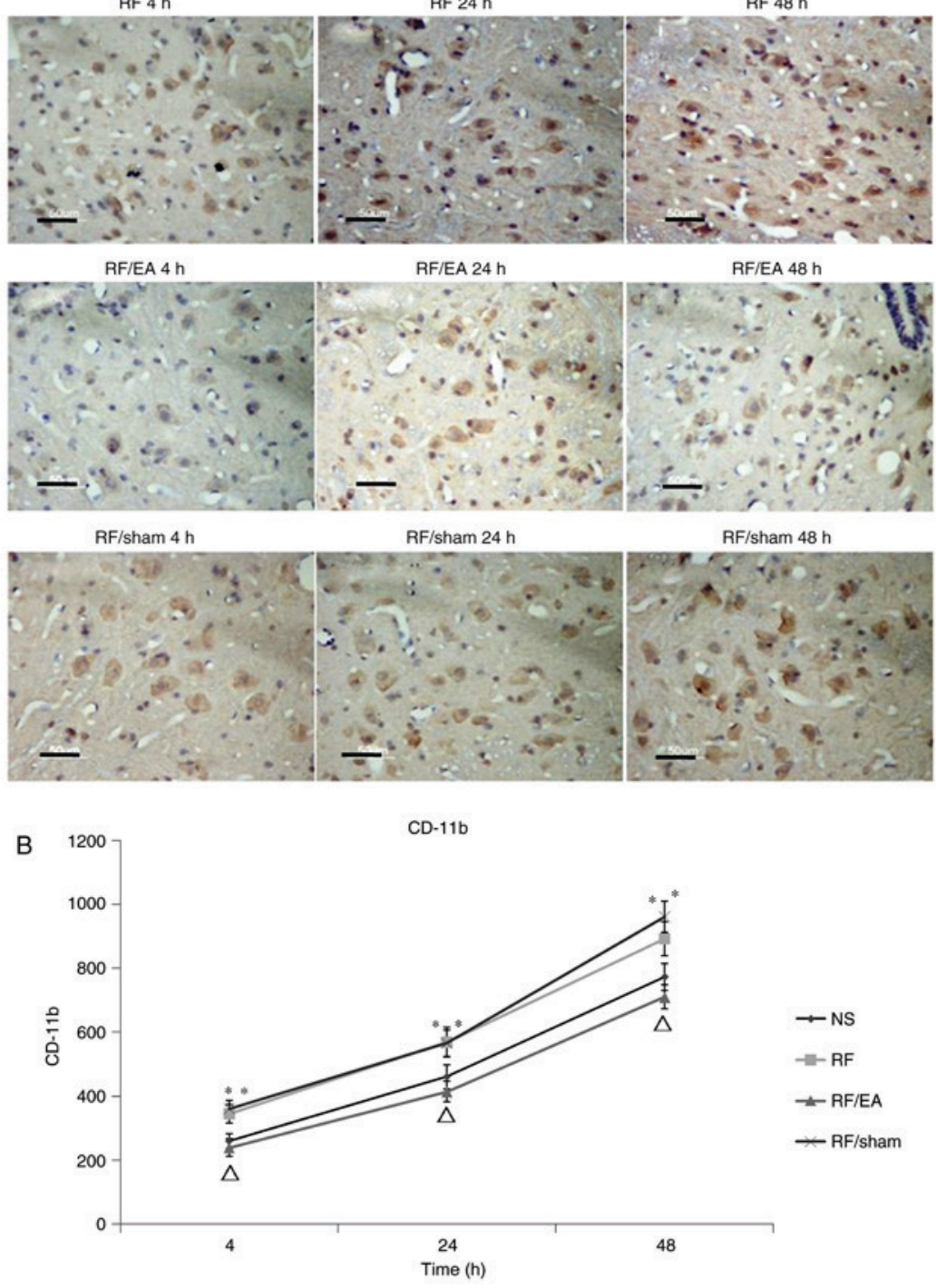

Figure 4. Effects of EA on CD-11b expression as assessed using immunohistochemistry. The L4-L5 segments of spinal cords were collected following behavioral testing. (A) Immunohistochemical images of each group at 4, 24 and $48 \mathrm{~h}$. Scale bar, $50 \mu \mathrm{m}$. (B) Mean CD-11b positive areas were quantified and assessed. ${ }^{* *} \mathrm{P}<0.05$ vs. NS and ${ }^{\Delta} \mathrm{P}<0.05$ vs. RF. EA, electro-acupuncture; RF, remifentanil; NS, normal saline; $\mathrm{CD}$, cluster of differentiation. 

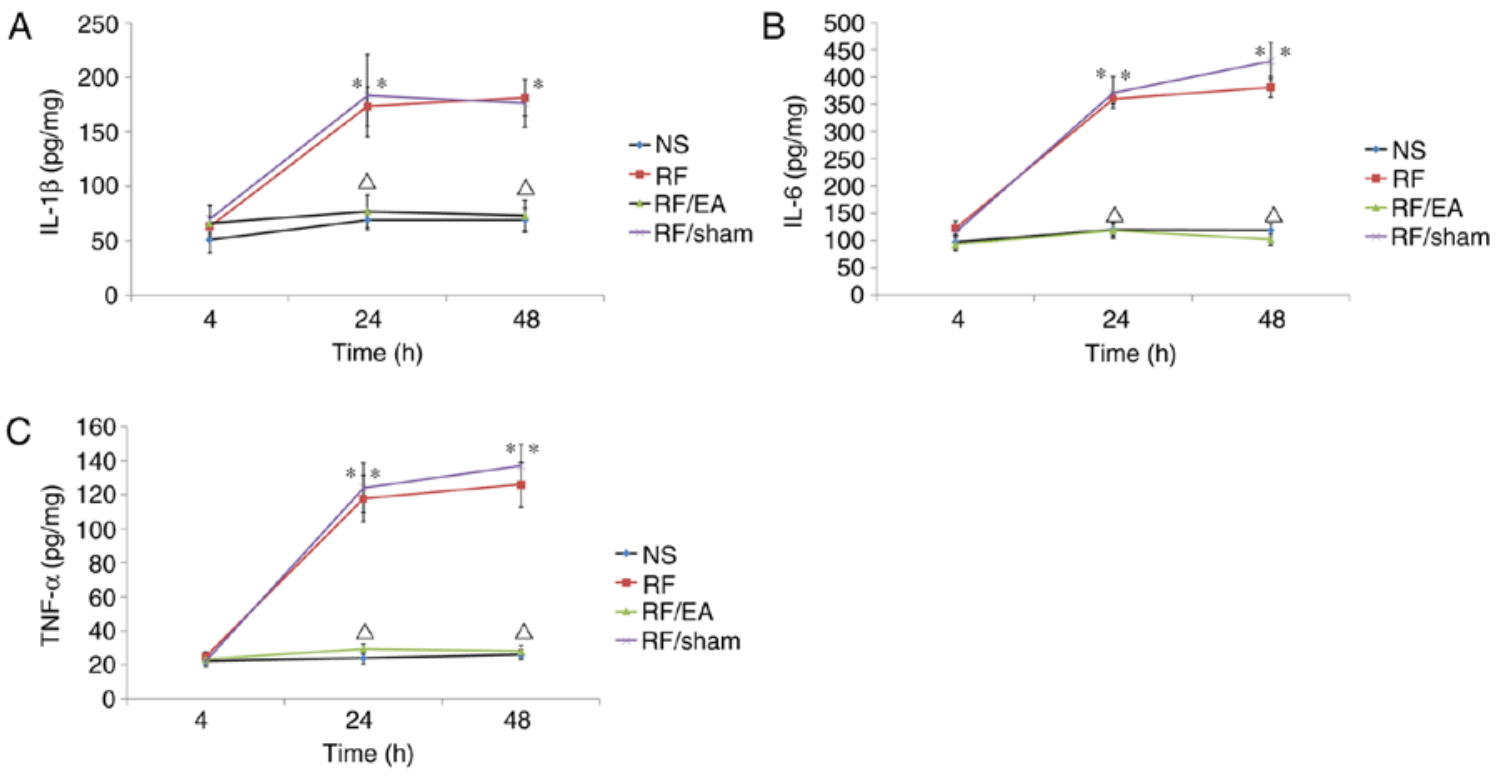

Figure 5. Effects of EA on (A) IL-1 $\beta$, (B) IL-6 and (C) TNF- $\alpha$ expression as assessed using ELISA. ${ }^{* *} \mathrm{P}<0.05$ vs. NS and ${ }^{\Delta} \mathrm{P}<0.05$ vs. RF. TNF, tumor necrosis factor; IL, interleukin; NS, normal saline; RF, remifentanil.

and RF/EA groups or between the RF and RF/sham groups at any time point. These results demonstrate that EA treatment is able to completely recover the number of spinal microglia following RF infusion, while sham EA has no significant effect.

EA decreases TNF- $\alpha, I L-1 \beta$ and IL- 6 levels. TNF- $\alpha$, IL-1 $\beta$ and IL-6 levels in the spinal cord at lumbar segments $\left(\mathrm{L}_{4-5}\right)$ were significantly higher in the RF and RF/EA-sham groups compared with the NS control group at 24 and $48 \mathrm{~h}$ following RF infusion $(\mathrm{P}<0.05$; Fig. 5), indicating that RF-induced hyperalgesia was associated with spinal inflammation. Furthermore, TNF- $\alpha$, IL-1 $\beta$ and IL- 6 levels in the RF/EA group were significantly reduced compared with those of the RF/EA-sham group at 24 and $48 \mathrm{~h}$ following infusion $(\mathrm{P}<0.05$; Fig. 5), which suggests that EA suppresses the inflammatory response in the spine. No significant differences were observed in TNF- $\alpha$, IL-1 $\beta$ or and IL-6 levels between the NS and RF/EA groups at any time point. These results suggest that EA completely suppresses the inflammatory response in the spine following RF infusion.

\section{Discussion}

In the present study, it was demonstrated that RF administration reduces the PWT and PWL values in rats, while the number CD11b positive cells is increased and TNF- $\alpha$, IL- $1 \beta$ and IL-6 are upregulated. These effects were significantly alleviated by treatment with EA at the Huantiao and Yanglingquan acupoints.

In the present study, RF was injected for $60 \mathrm{~min}$ to construct a rat model of RIPH. Reduced PWT and PWL values confirmed that the model had been successfully established. Cooper et al (26) reported that the area of mechanical hyperalgesia is significantly extended for $30 \mathrm{~min}$ following the end of RF infusion for $90 \mathrm{~min}$. It has also been reported that RIPH occurs $2 \mathrm{~h}$ after anesthesia, peaking at 24-48 h (34). In addition, Celerier et al (35) demonstrated that $0.04 \mathrm{mg} / \mathrm{kg}$ RF-induced hyperalgesia occurs at $24 \mathrm{~h}$ and peaks at $24-48 \mathrm{~h}$ post-surgery. These studies corroborate those of the present study.
According to Traditional Chinese Medicine, acupuncture at the Huantiao and Yanglingquan acupoints is effective for the treatment of sciatica (36). EA has been used successfully in patients treated with RF for pain relief, both postoperatively $(22,37,38)$ and during surgery (39). It has has been demonstrated that administering EA 30 min before anesthesia improves cognitive function postoperatively, with reduced inflammation (40).

In the present study, PWT and PWL values were higher in the RF/EA group compared with the RF/EA-sham group, with no significant differences observed between the RF/EA and NS groups, indicating that electrical stimulation at the Huantiao and Yanglingquan acupoints significantly alleviated RIPH. These findings corroborate a previous study in which it was demonstrated that RIPH decreases mechanical stimuli required and the thermal pain threshold around the incision (41). The results are consistent with a previous study by our group in which it was demonstrated that EA alleviates postoperative pain in patients undergoing thoracic esophagectomy (22).

The underlying mechanism responsible for the action of EA in RF-induced hypoanalgesia remains to be elucidated. EA at acupoints may release endogenous analgesics, including opioid peptides, adenosine and 5-hydroxytryptamine (23-25,32). EA at the Huantiao and Yanglingquan acupoints decreased the number of microglia and suppressed the RF-induced inflammatory response in the spinal cord. These findings suggest that EA likely alleviates RIPH by suppressing activated spinal colloid cells that release large amounts of proinflammatory cytokines. Using this as a basis, specific targeting of microglia may be an effective method for reducing postoperative pain and deserves further attention.

The main limitation of the present study is that it was performed in a rat model, which may not translate exactly to humans. However, rat acupoints do correspond with human acupoints to a certain degree in terms of anatomy and physiological functions (42-44). Previously studies have used pathological rat models to assess the curative effects of acupuncture (42-44). In the present study, EA was 
demonstrated to have curative effects when used to stimulate specific acupuncture points in a rat model, which suggests that these acupoints have a similar regulatory effect to those in humans. Nevertheless, animal experiments are only intended to provide a tentative exploration of possible mechanisms and these hypotheses remain to be further explored in humans.

In summary, the results of the present preliminary study demonstrate that EA inhibits RIPH in an incision pain rat model, likely by decreasing the number of activated microglia in the spinal cord and therefore reducing the expression of proinflammatory cytokines. As such, controlling the activation of spinal microglia may be a novel method for managing postoperative pain.

\section{Acknowledgements}

The authors would like to thank Professor Liecheng Wang at the Department of Physiology, Anhui Medical University.

\section{Funding}

The present study was supported by the Department of Health of Anhui Province (grant no. 2012zy45).

\section{Availability of data and materials}

The datasets used or analyzed during the current study are available from the corresponding author on reasonable request.

\section{Authors' contributions}

YX, JM and DI conceived and designed the experiments. YX and JM performed the experiments. YX and $\mathrm{CG}$ analyzed the data. DI and XC revised the manuscript and approved the final version.

\section{Ethics approval and consent to participate}

The experimental protocols were approved by the Institutional Animal Experimental Ethics Committee of Anhui Medical University (Hefei, China). All procedures were performed in accordance with the ethical standards of the Institutional Animal Care and Use Committee of Anhui Medical University.

\section{Consent for publication}

Not applicable.

\section{Competing interests}

The authors declare that they have no competing interests.

\section{References}

1. Khanykin B, Siddiqi R, Jensen PF, Bigler DR and Atroshchenko GV: Comparison of remifentanil and low-dose fentanyl for fast-track cardiac anesthesia: A prospective randomized study. Heart Surg Forum 16: E324-328, 2013.

2. Douma MR, Verwey RA, Kam-Endtz CE, van der Linden PD and Stienstra R: Obstetric analgesia: A comparison of patient-controlled meperidine, remifentanil, and fentanyl in labour. Br J Anaesth 104: 209-215, 2010.
3. Fletcher D and Martinez V: Opioid-induced hyperalgesia in patients after surgery: A systematic review and a meta-analysis. Br J Anaesth 112: 991-1004, 2014.

4. Rivosecchi RM, Rice MJ, Smithburger PL, Buckley MS, Coons JC and Kane-Gill SL: An evidence based systematic review of remifentanil associated opioid-induced hyperalgesia. Expert Opin Drug Saf 13: 587-603, 2014.

5. Petrenko AB, Ishii $\mathrm{H}$, Kohno $\mathrm{T}$ and Baba $\mathrm{H}$ : When similar is not alike: Decreased sensory thresholds after intravenous infusion of remifentanil may not be remifentanil-induced hyperalgesia. Anesth Analg 115: 977, 2012.

6. Berta T, Park CK, Xu ZZ, Xie RG, Liu T, Lü N, Liu YC and Ji RR: Extracellular caspase-6 drives murine inflammatory pain via microglial TNF- $\alpha$ secretion. J Clin Invest 124: 1173-1186, 2014.

7. Huang CT, Chiang RP, Chen CL and Tsai YJ: Sleep deprivation aggravates median nerve injury-induced neuropathic pain and enhances microglial activation by suppressing melatonin secretion. Sleep 37: 1513-1523, 2014

8. Mika J, Popiolek-Barczyk K, Rojewska E, Makuch W, Starowicz K and Przewlocka B: Delta-opioid receptor analgesia is independent of microglial activation in a rat model of neuropathic pain. PloS One 9: e104420, 2014.

9. Vickers AJ, Rusch VW, Malhotra VT, Downey RJ and Cassileth BR: Acupuncture is a feasible treatment for post-thoracotomy pain: Results of a prospective pilot trial. BMC Anesthesiol 6: 5, 2006.

10. Milligan ED, Twining C, Chacur M, Biedenkapp J, O'Connor K, Poole S, Tracey K, Martin D, Maier SF and Watkins LR: Spinal glia and proinflammatory cytokines mediate mirror-image neuropathic pain in rats. J Neurosci 23: 1026-1040, 2003.

11. Zhang L, Berta T, Xu ZZ, Liu T, Park JY and Ji RR: TNF- $\alpha$ contributes to spinal cord synaptic plasticity and inflammatory pain: Distinct role of TNF receptor subtypes 1 and 2. Pain 152: 419-427, 2011.

12. Maresz K, Pryce G, Ponomarev ED, Marsicano G, Croxford JL, Shriver LP, Ledent C, Cheng X, Carrier EJ, Mann MK, et al: Direct suppression of CNS autoimmune inflammation via the cannabinoid receptor $\mathrm{CB} 1$ on neurons and $\mathrm{CB} 2$ on autoreactive $\mathrm{T}$ cells. Nat Med 13: 492-497, 2007.

13. Sun Y, Zhang W, Liu Y, Liu X, Ma Z and Gu X: Intrathecal injection of JWH015 attenuates remifentanil-induced postoperative hyperalgesia by inhibiting activation of spinal glia in a rat model. Anesth Analg 118: 841-853, 2014.

14. Song JW, Lee YW, Yoon KB, Park SJ and Shim YH: Magnesium sulfate prevents remifentanil-induced postoperative hyperalgesia in patients undergoing thyroidectomy. Anesth Analg 113: 390-397, 2011.

15. Echevarria G, Elgueta F, Fierro C, Bugedo D, Faba G, Iñiguez-Cuadra R, Muñoz HR and Cortínez LI: Nitrous oxide $(\mathrm{N}(2) \mathrm{O})$ reduces postoperative opioid-induced hyperalgesia after remifentanil-propofol anaesthesia in humans. Br J Anaesth 107: 959-965, 2011.

16. Elterman KG, Mallampati SR, Kaye AD and Urman RD: Postoperative alterations in taste and smell. Anesth Pain Med 4: e18527, 2014.

17. Chen T, Wang K, Xu J, Ma W and Zhou J: Electroacupuncture reduces postoperative pain and analgesic consumption in patients undergoing thoracic surgery: A randomized study. Evid Based Complement Alternat Med 2016: 2126416, 2016.

18. Linde K, Vickers A, Hondras M, ter Riet G, Thormählen J, Berman B and Melchart D: Systematic reviews of complementary therapies - an annotated bibliography. Part 1: Acupuncture. BMC Complement Altern Med 1: 3, 2001.

19. Park JH, Han JB, Kim SK, Park JH, Go DH, Sun B and Min BI: Spinal GABA receptors mediate the suppressive effect of electroacupuncture on cold allodynia in rats. Brain Res 1322: 24-29, 2010.

20. Ma W, Zhu YM,Zhou H, Fu GQ, Pan H and Shen WD: Protecting action of acupuncture-drug compound anesthesia with different frequency electroacupuncture on stress reaction in pneumonectomy. Zhongguo Zhen Jiu 31: 1020-1024, 2011 (In Chinese).

21. Robinson CR, Zhang H and Dougherty PM: Astrocytes, but not microglia, are activated in oxaliplatin and bortezomib-induced peripheral neuropathy in the rat. Neuroscience 274: 308-317, 2014.

22. Xie YH, Chai XQ, Wang YL, Gao YC and Ma J: Effect of electro-acupuncture stimulation of Ximen (PC4) and Neiguan (PC6) on remifentanil-induced breakthrough pain following thoracal esophagectomy. J Huazhong Univ Sci Technolog Med Sci 34: 569-574, 2014. 
23. Su TF, Zhang LH, Peng M, Wu CH, Pan W, Tian B, Shi J, Pan HL and Li M: Cannabinoid CB2 receptors contribute to upregulation of $\beta$-endorphin in inflamed skin tissues by electroacupuncture. Mol Pain 7: 98, 2011.

24. Fais RS, Reis GM, Silveira JW, Dias QM, Rossaneis AC and Prado WA: Amitriptyline prolongs the antihyperalgesic effect of 2- or $100-\mathrm{Hz}$ electro-acupuncture in a rat model of post-incision pain. Eur J Pain 16: 666-675, 2012.

25. Onda A, Jiao Q, Nagano Y, Akimoto T, Miyamoto T, Minamisawa S and Fukubayashi T: Acupuncture ameliorated skeletal muscle atrophy induced by hindlimb suspension in mice. Biochem Biophys Res Commun 410: 434-439, 2011.

26. Cooper ZD, Truong YN, Shi YG and Woods JH: Morphine deprivation increases self-administration of the fast- and short-acting mu-opioid receptor agonist remifentanil in the rat. J Pharmacol Exp Ther 326: 920-929, 2008

27. Hanisch UK and Kettenmann H: Microglia: Active sensor and versatile effector cells in the normal and pathologic brain. Nat Neurosci 10: 1387-1394, 2007.

28. 28. Hua X, LI C, Zhou H, Song D, Hu Y: The trituration of the atlas of the rat acupoints. Shiyan Dongwu Yu Dongwu Shiyan 3 : $1-5,1991$

29. Romita VV, Suk A and Henry JL: Parametric studies on electroacupuncture-like stimulation in a rat model: Effects of intensity, frequency, and duration of stimulation on evoked antinociception. Brain Res Bull 42: 289-296, 1997.

30. Lao L, Zhang RX, Zhang G, Wang X, Berman BM and Ren K: A parametric study of electroacupuncture on persistent hyperalgesia and Fos protein expression in rats. Brain Res 1020: 18-29, 2004.

31. Fang JL, Krings T, Weidemann J, Meister IG and Thron A: Functional MRI in healthy subjects during acupuncture: Different effects of needle rotation in real and false acupoints. Neuroradiology 46: 359-362, 2004.

32. Brennan TJ, Vandermeulen EP and Gebhart GF: Characterization of a rat model of incisional pain. Pain 64: 493-501, 1996.

33. Yuan Y, Wang JY, Yuan F, Xie KL, Yu YH and Wang GL: Glycogen synthase kinase- $3 \beta$ contributes to remifentanil-induced postoperative hyperalgesia via regulating $\mathrm{N}$-methyl-D-aspartate receptor trafficking. Anesth Analg 116: 473-481, 2013.

34. Gu X, Wu X, Liu Y, Cui S and Ma Z: Tyrosine phosphorylation of the N-Methyl-D-Aspartate receptor 2B subunit in spinal cord contributes to remifentanil-induced postoperative hyperalgesia: The preventive effect of ketamine. Mol Pain 5: 76, 2009.

35. Celerier E, Gonzalez JR, Maldonado R, Cabanero D and Puig MM: Opioid-induced hyperalgesia in a murine model of postoperative pain: Role of nitric oxide generated from the inducible nitric oxide synthase. Anesthesiology 104: 546-555, 2006.
36. Ji M, Wang X, Chen M, Shen Y, Zhang X and Yang J: The efficacy of acupuncture for the treatment of sciatica: A systematic review and meta-analysis. Evid Based Complement Alternat Med 2015: 192808, 2015

37. Iacobone M, Citton M,Zanella S, Scarpa M, Pagura G, Tropea S, Galligioni H, Ceccherelli F, Feltracco P, Viel G and Nitti D: The effects of acupuncture after thyroid surgery: A randomized, controlled trial. Surgery 156: 1605-1612, 2014.

38. Wang H, Xie Y, Zhang Q, Xu N, Zhong H, Dong H, Liu L, Jiang T, Wang Q and Xiong L: Transcutaneous electric acupoint stimulation reduces intra-operative remifentanil consumption and alleviates postoperative side-effects in patients undergoing sinusotomy: A prospective, randomized, placebo-controlled trial. Br JAnaesth 112: 1075-1082, 2014

39. Sator-Katzenschlager SM, Wolfler MM, Kozek-Langenecker SA, Sator K, Sator PG, Li B, Heinze G and Sator MO: Auricular electro-acupuncture as an additional perioperative analgesic method during oocyte aspiration in IVF treatment. Hum Reprod 21: 2114-2120, 2006.

40. Zhang Q, Li YN, Guo YY, Yin CP, Gao F, Xin X, Huo SP, Wang XL and Wang QJ: Effects of preconditioning of electro-acupuncture on postoperative cognitive dysfunction in elderly: A prospective, randomized, controlled trial. Medicine (Baltimore) 96: e7375, 2017.

41. Zhao Mand JooDT: Enhancement of spinalN-methyl-D-aspartate receptor function by remifentanil action at delta-opioid receptors as a mechanism for acute opioid-induced hyperalgesia or tolerance. Anesthesiology 109: 308-317, 2008

42. Zhang Y, Zhang RX, Zhang M, Shen XY, Li A, Xin J, Ren K, Berman BM, Tan M and Lao L: Electroacupuncture inhibition of hyperalgesia in an inflammatory pain rat model: Involvement of distinct spinal serotonin and norepinephrine receptor subtypes. Br J Anaesth 109: 245-252, 2012.

43. Liu W, Wu J, Huang J, Zhuo P, Lin Y, Wang L, Lin R, Chen L and Tao J: Electroacupuncture regulates hippocampal synaptic plasticity via miR-134-Mediated LIMK1 function in rats with ischemic stroke. Neural Plast 2017: 9545646, 2017.

44. Zhu Y, Deng L, Tang H, Gao X, Wang Y, Guo K, Kong J and Yang C: Electroacupuncture improves neurobehavioral function and brain injury in rat model of intracerebral hemorrhage. Brain Res Bull 131: 123-132, 2017. 